\title{
Approximate Bound State Solutions for Certain Molecular Potentials
}

\author{
Mahmoud Farout ${ }^{1 *}$, Mohammed Yasin ${ }^{2 *}$, Sameer M. Ikhdair ${ }^{1,3}$ \\ ${ }^{1}$ Department of Physics, An-Najah National University, Nablus, Palestine \\ ${ }^{2}$ Department of Mathematics, An-Najah National University, Nablus, Palestine \\ ${ }^{3}$ Department of Electrical Engineering, Near East University, Nicosia, Northern Cyprus, Turkey \\ Email: ^m.qaroot@najah.edu, ^m.yasin@najah.edu, sameer.ikhdair@najah.edu
}

How to cite this paper: Farout, M., Yasin, M. and Ikhdair, S.M. (2021) Approximate Bound State Solutions for Certain Molecular Potentials. Journal of Applied Mathematics and Physics, 9, 736-750. https://doi.org/10.4236/jamp.2021.94052

Received: March 12, 2021

Accepted: April 25, 2021

Published: April 28, 2021

Copyright (c) 2021 by author(s) and Scientific Research Publishing Inc. This work is licensed under the Creative Commons Attribution International License (CC BY 4.0).

http://creativecommons.org/licenses/by/4.0/ (c) (i) Open Access

\begin{abstract}
We present solutions of the Schrodinger equation with superposition of Manning-Rosen plus inversely Mobius square plus quadratic Yukawa potentials using parametric Nikiforov Uvarov method along with an approximation to the centrifugal term. The bound state energy eigenvalues for any angular momentum quantum number $l$ and the corresponding un-normalized wave functions are calculated. The mixed potential which in some particular cases gives the solutions for different potentials: the Manning-Rosen, the Mobius square, the inversely quadratic Yukawa and the Hulthén potentials along with their bound state energies are obtained.
\end{abstract}

\section{Keywords}

Schrödinger Equation, Mobius Potential, Manning-Rosen Potential, Quadratic Yukawa Potential, Hulthén Potential, Bound State Energies, Wave Functions

\section{Introduction}

The Schrödinger wave equation is primarily considered as one of the most commonly used differential equations in non-relativistic quantum mechanics [1] [2] [3]. However, since the early times of quantum mechanics, the exact solutions of the Schrödinger equation with some particular physical potentials are of much interest. Such solutions provide profound conceptual understanding to physical models and certainly lead to a strong judgment supporting the correctness of quantum theory. The exact solutions of central and non-central potentials find their applications in various branches of physics such as molecular, solid-state and chemical physics [4] and so forth. Our choice for the real poten- 
tial gives the bound state energy eigenvalues and wave functions of the Schrödinger wave equation that might describe essentially the particle dynamics in non-relativistic quantum mechanics. Moreover, these solutions are used in checking and try to improve models under study and then also finding methods in solving complicated physical models.

Since the early times of quantum mechanics the number of exactly solvable physical problems is very limited. Several authors have paid many efforts toward studying the exactly solvable physical problems by which one can determine the whole energy spectrum analytically for wide range values of potential parameters [5]. Therefore, in most of these potentials, the quasi-exactly solvable potentials are the ones that provide a part of the energy spectrum [6].

Recently, various methods are introduced and employed in quantum mechanics in solving the wave equations with a particular given solvable potential. We mention few among the many methods: the group theoretical technique [7], the factorization method [8] [9], functional analysis approach (FAA) [10], supersymmetric (SUSY) quantum mechanics [11], shape invariance (SI) [12], the Nikiforov-Uvarov (NU) method [13], exact quantization rule [14] [15] and asymptotic iteration method (AIM) [16].

The Manning-Rosen, the quadratic Yukawa and the Mobius square potentials have been intensively considered and studied in non-relativistic and relativistic wave equations in recent years [17]-[27]. Therefore, the main motivation of the present work is to give approximate solution to the non-relativistic Schrödinger equation with the superposition of Manning-Rosen plus inversely Mobius square plus Yukawa potential models. Hence we need to treat the centrifugal term with Greene-Aldrich approximation to enable for analytical solution of the Schrödinger equation for any angular momentum quantum number 1 . This would provide us the bound state energy spectrum for any angular momentum quantum number $l$ and the corresponding wave functions by simply applying the parametric Nikiforov-Uvarov (pNU) method.

The structure of the present work is as follows. In Section 2, we present the brief methodology. In Section 3, we apply this method to derive the bound state energy and wave functions for the Schrödinger equation with the present potential model. Section 4 presents our results and discussion. Finally, in Section 5, we give our conclusion.

\section{Methodology}

The Nikiforov-Uvarov (NU) [13] method is an efficient tool which is usually used to reduce the second-order differential equation into a general form of a hypergeometric type. In that sense, any second order differential equation, i.e. Schrödinger, Fienberg-Horodecki [28] [29] [30] [31], relativistic Dirac, Klien-Gordon equation, etc., can be transformed, using a suitable coordinate transformation $s$ $=s(t)$, into the form:

$$
\psi_{n}^{\prime \prime}(s)+\frac{\tilde{\tau}(s)}{\sigma(s)} \psi_{n}^{\prime}(s)+\frac{\tilde{\sigma}(s)}{\sigma^{2}(s)} \psi_{n}(s)=0,
$$


where $\sigma(s)$ and $\tilde{\sigma}(s)$ are polynomials, at most second-degree, and $\tilde{\tau}(s)$ is a first-degree polynomial. The method is noted to be tiresome and time-consuming. Therefore, Tezcan and Sever [32] derived a parametric form of the NU method to popularize the method more. The parametric NU method is straightforward, simpler and more accurate for the determination of the energy eigenvalues and the corresponding eigenstates. To apply the parametric NU method, the differential equation must be set into the general form given by [33]

$$
\psi_{n}^{\prime \prime}(s)+\frac{\alpha_{1}-\alpha_{2} s}{s\left(1-\alpha_{3} s\right)} \psi_{n}^{\prime}(s)+\frac{-\gamma_{1}^{2} s^{2}+\gamma_{2} s-\gamma_{3}}{s^{2}\left(1-\alpha_{3} s\right)^{2}} \psi_{n}(s)=0,
$$

The conditions for the energy eigenvalues and the corresponding eigenstates are, respectively, given as

$$
\begin{aligned}
& \left(\alpha_{2}-\alpha_{3}\right) n+\alpha_{3} n^{2}-(2 n+1) \alpha_{5}+(2 n+1)\left(\sqrt{\alpha_{9}}+\alpha_{3} \sqrt{\alpha_{8}}\right) \\
& +\alpha_{7}+2 \alpha_{3} \alpha_{8}+2 \sqrt{\alpha_{8} \alpha_{9}}=0, \\
& \psi_{n}(s)=N_{n l} s^{\alpha_{12}}\left(1-\alpha_{3} s\right)^{-\alpha_{12}-\left(\alpha_{13} / \alpha_{3}\right)} P_{n}^{\left(\alpha_{10}-1, \frac{\alpha_{11}}{\alpha_{3}}-\alpha_{10}-1\right)}\left(1-2 \alpha_{3} s\right),
\end{aligned}
$$

where

$$
\begin{gathered}
\alpha_{4}=\frac{1}{2}\left(1-\alpha_{1}\right), \\
\alpha_{5}=\frac{1}{2}\left(\alpha_{2}-2 \alpha_{3}\right), \\
\alpha_{6}=\alpha_{5}^{2}+\gamma_{1}, \\
\alpha_{7}=2 \alpha_{4} \alpha_{5}-\gamma_{2}, \\
\alpha_{8}=\alpha_{4}^{2}+\gamma_{3}, \alpha_{9}=\alpha_{3} \alpha_{7}+\alpha_{3}^{2} \alpha_{8}+\alpha_{6}, \\
\alpha_{10}=\alpha_{1}+2 \alpha_{4}+2 \sqrt{\alpha_{8}}, \\
\alpha_{11}=\alpha_{2}-2 \alpha_{5}+2\left(\sqrt{\alpha_{9}}+\alpha_{3} \sqrt{\alpha_{8}}\right), \\
\alpha_{12}=\alpha_{4}+\sqrt{\alpha_{8}}, \\
\alpha_{13}=\alpha_{5}-\left(\sqrt{\alpha_{9}}+\alpha_{3} \sqrt{\alpha_{8}}\right),
\end{gathered}
$$

where $N_{n l}$ is the normalisation constant and $P_{n}^{(\beta, \gamma)}$ is the orthogonal Jacobi polynomial.

\section{Solution of the Schrödinger Equation with Two Molecular Potential Models}

The Schrödinger equation in spherical coordinates is given as [33]

$$
\begin{aligned}
& -\frac{\hbar^{2}}{2 \mu}\left[\frac{1}{r^{2}} \frac{\partial}{\partial r}\left(r^{2} \frac{\partial}{\partial r}\right)+\frac{1}{r^{2} \sin \theta} \frac{\partial}{\partial \theta}\left(\sin \theta \frac{\partial}{\partial \theta}\right)+\frac{1}{r^{2} \sin ^{2} \theta} \frac{\partial^{2}}{\partial \phi^{2}}\right] \psi(r, \theta, \phi) \\
& =E \psi(r, \theta, \phi),
\end{aligned}
$$

where $\hbar$ is the reduced Plank constant, $\mu$ is the reduced mass, $E$ is the ener- 
gy eigenvalues, and $\psi$ is the wave function of the particle. If we define the wave function as

$$
\psi(r, \theta, \phi)=\frac{R_{n l}(r)}{r} Y_{l m}(\theta, \phi),
$$

the radial part of Schrödinger equation is given by

$$
\frac{\mathrm{d}^{2} R_{n l}(r)}{\mathrm{d} r^{2}}+\left[\frac{2 \mu}{\hbar^{2}}(E-V(r))-\frac{l(l+1)}{r^{2}}\right] R_{n l}(r)=0,
$$

where $n$ and $l$ are the radial and the angular momentum quantum numbers, respectively.

We shall solve the Schrodinger equation for the following two molecular potential models:

\subsection{Combination of Manning-Rosen Plus Mobius Square Plus Quadratic Yukawa Potentials}

The general potential is given as [34] [35] [36]

$$
V(r)=-\left(\frac{C \mathrm{e}^{-\alpha r}+D \mathrm{e}^{-2 \alpha r}}{\left(1-\mathrm{e}^{-\alpha r}\right)^{2}}\right)-V_{0}\left(\frac{A+B \mathrm{e}^{-\alpha r}}{1-\mathrm{e}^{-\alpha r}}\right)^{2}+\frac{V_{1} \mathrm{e}^{-\alpha r}}{r^{2}},
$$

where $C, D, V_{0}, V_{1}$ are potential parameters and $\alpha$ is the screening parameter.

It is obvious that Equation (16) cannot be solved analytically due to the quadratic Yukawa and the centrifugal terms. However, this can be addressed using the Green-Aldrich approximation [37]

$$
\frac{1}{r^{2}} \approx \frac{\alpha^{2} \mathrm{e}^{-\alpha r}}{\left(1-\mathrm{e}^{-\alpha r}\right)^{2}} .
$$

Substituting Equation (17) into Equation (16) and using Equation (18) with $s=\mathrm{e}^{-\alpha r}$ one obtains Equation (2), where

$$
\begin{gathered}
-\gamma_{1}^{2}=\frac{2 \mu}{\hbar^{2} \alpha^{2}}\left(E+D+V_{0} B^{2}-\alpha^{2} V_{1}\right), \\
\gamma_{2}=\frac{2 \mu}{\hbar^{2} \alpha^{2}}\left(C+2 A B V_{0}-2 E\right)-l(l+1), \\
\gamma_{3}=-\frac{2 \mu}{\hbar^{2} \alpha^{2}}\left(E+V_{0} A^{2}\right) .
\end{gathered}
$$

Comparing Equation (38) with the parameters Equations (5) to (13), one gets

$$
\begin{gathered}
\alpha_{1}=\alpha_{2}=\alpha_{3}=1, \alpha_{4}=0, \alpha_{5}=-\frac{1}{2}, \\
\alpha_{6}=\frac{1}{4}-\frac{2 \mu}{\hbar^{2} \alpha^{2}}\left(E+D+V_{0} B^{2}-\alpha^{2} V_{1}\right), \\
\alpha_{7}=-\frac{2 \mu}{\hbar^{2} \alpha^{2}}\left(C+2 A B V_{0}-2 E\right)-l(l+1), \\
\alpha_{8}=-\frac{2 \mu}{\hbar^{2} \alpha^{2}}\left(E+V_{0} A^{2}\right),
\end{gathered}
$$




$$
\begin{gathered}
\alpha_{9}=\frac{1}{4}(2 l+1)^{2}-\frac{2 \mu}{\hbar^{2} \alpha^{2}}\left(C+D+(A+B)^{2} V_{0}-\alpha^{2} V_{1}\right) \\
\alpha_{10}=1+2 \sqrt{-\frac{2 \mu}{\hbar^{2} \alpha^{2}}\left(E+V_{0} A^{2}\right)} \\
\alpha_{11}=2+2\left[\frac{1}{2} \sqrt{(2 l+1)^{2}-\frac{8 \mu}{\hbar^{2} \alpha^{2}}\left(C+D+(A+B)^{2} V_{0}-\alpha^{2} V_{1}\right)}\right. \\
\left.+\sqrt{-\frac{2 \mu}{\hbar^{2} \alpha^{2}}\left(E+V_{0} A^{2}\right)}\right] \\
\alpha_{13}=-\frac{1}{2}-\left[\frac{1}{2} \sqrt{(2 l+1)^{2}-\frac{8 \mu}{\hbar^{2} \alpha^{2}}\left(C+D+(A+B)^{2} V_{0}-\alpha^{2} V_{1}\right)}+\sqrt{-\frac{2 \mu E}{\hbar^{2} \alpha^{2}}}\right]
\end{gathered}
$$

Substituting the values of the parametric constants Equations (22) to (30) into Equations (3) and (4), respectively, one gets the energy eigenvalues and the corresponding unnormalized radial eigenstates as

$$
E_{n l}=-V_{0} A^{2}-\frac{\hbar^{2} \alpha^{2}}{2 \mu}\left[\frac{n(n+1)+l(l+1)+\frac{1}{2}+(n+1) T-\frac{2 \mu}{\hbar^{2} \alpha^{2}}\left(C+2 V_{0} A(A+B)\right)}{1+2 n+T}\right]^{2},
$$

and

$$
\psi_{n l}(r)=N_{n l} \mathrm{e}^{-\alpha \sqrt{\gamma_{3}} r}\left(1-\mathrm{e}^{-\alpha r}\right)^{\beta} P_{n}^{\left(2 \sqrt{\gamma_{3}}, T\right)}\left(1-2 \mathrm{e}^{-\alpha r}\right),
$$

where

$$
\begin{gathered}
T=\sqrt{(2 l+1)^{2}-\frac{8 \mu}{\hbar^{2} \alpha^{2}}\left(C+D+(A+B)^{2} V_{0}-\alpha^{2} V_{1}\right)}, \\
\gamma_{3}=-\frac{2 \mu}{\hbar^{2} \alpha^{2}}\left(E+V_{0} A^{2}\right),
\end{gathered}
$$

and $\beta=\frac{1+T}{2}-\sqrt{\gamma_{3}}$.

\subsection{Combination of Manning-Rosen Plus Quadratic Yukawa Potentials}

The Manning-Rosen plus quadratic Yukawa potential is given by [34] [35]

$$
V(r)=-\left[\frac{C \mathrm{e}^{-\alpha r}+D \mathrm{e}^{-2 \alpha r}}{\left(1-\mathrm{e}^{-\alpha r}\right)^{2}}\right]+\frac{V_{1} \mathrm{e}^{-\alpha r}}{r^{2}},
$$

where $C, D, V_{1}$ are potential parameters and $\alpha$ is the screening parameter.

Substituting Equation (35) into Equation (16) gives

$$
\frac{\mathrm{d}^{2} R_{n l}(r)}{\mathrm{d} r^{2}}+\left[\frac{2 \mu}{\hbar^{2}}\left(E+\left(\frac{C \mathrm{e}^{-\alpha r}+D \mathrm{e}^{-2 \alpha r}}{\left(1-\mathrm{e}^{-\alpha r}\right)^{2}}\right)-\frac{V_{1} \mathrm{e}^{-\alpha r}}{r^{2}}\right)-\frac{l(l+1)}{r^{2}}\right] R_{n l}(r)=0 .
$$


Substituting Equation (18) in Equation (36) leads

$\frac{\mathrm{d}^{2} R_{n l}(r)}{\mathrm{d} r^{2}}+\left[\frac{2 \mu}{\hbar^{2}}\left(E+\left(\frac{C \mathrm{e}^{-\alpha r}+D \mathrm{e}^{-2 \alpha r}}{\left(1-\mathrm{e}^{-\alpha r}\right)^{2}}\right)-\frac{V_{1} \alpha^{2} \mathrm{e}^{-2 \alpha r}}{\left(1-\mathrm{e}^{-\alpha r}\right)^{2}}\right)-\frac{l(l+1) \alpha^{2} \mathrm{e}^{-\alpha r}}{\left(1-\mathrm{e}^{-\alpha r}\right)^{2}}\right] R_{n l}(r)=0$.

Now, changing of variables using $s=\mathrm{e}^{-\alpha r}$ to transform the equation to the form of Equation (2), one obtains

$$
R_{n l}^{\prime \prime}(s)+\frac{1-s}{s(1-s)} R_{n l}^{\prime}(s)+\frac{-\gamma_{1}^{2} s^{2}+\gamma_{2} s-\gamma_{3}}{s^{2}(1-s)^{2}} R_{n l}(s)=0,
$$

where

$$
\begin{gathered}
-\gamma_{1}^{2}=\frac{2 \mu}{\hbar^{2} \alpha^{2}}\left(E+D-\alpha^{2} V_{1}\right), \\
\gamma_{2}=\frac{2 \mu}{\hbar^{2} \alpha^{2}}(C-2 E)-l(l+1), \\
\gamma_{3}=-\frac{2 \mu E}{\hbar^{2} \alpha^{2}} .
\end{gathered}
$$

Comparing Equation (38) with the parameters Equations (5) to (13), one gets

$$
\begin{gathered}
\alpha_{1}=\alpha_{2}=\alpha_{3}=1, \alpha_{4}=0, \alpha_{5}=-\frac{1}{2} \\
\alpha_{6}=\frac{1}{4}-\frac{2 \mu}{\hbar^{2} \alpha^{2}}\left(E+D-\alpha^{2} V_{1}\right), \\
\alpha_{7}=-\frac{2 \mu}{\hbar^{2} \alpha^{2}}(C-2 E)-l(l+1), \\
\alpha_{8}=-\frac{2 \mu E}{\hbar^{2} \alpha^{2}}, \\
\alpha_{9}=\frac{1}{4}(2 l+1)^{2}-\frac{2 \mu}{\hbar^{2} \alpha^{2}}\left(C+D-\alpha^{2} V_{1}\right), \\
\alpha_{10}=1+2 \sqrt{-\frac{2 \mu E}{\hbar^{2} \alpha^{2}}}, \\
\alpha_{12}=\sqrt{\left.\frac{1}{2} \sqrt{(2 l+1)^{2}-\frac{8 \mu}{\hbar^{2} \alpha^{2}}\left(C+D-\alpha^{2} V_{1}\right)}+\sqrt{-\frac{2 \mu E}{\hbar^{2} \alpha^{2}}}\right],} \\
\alpha_{13}=-\frac{1}{2}-\left[\frac{1}{2} \sqrt{(2 l+1)^{2}-\frac{8 \mu}{\hbar^{2} \alpha^{2}}\left(C+D-\alpha^{2} V_{1}\right)}+\sqrt{-\frac{2 \mu E}{\hbar^{2} \alpha^{2}}}\right] .
\end{gathered}
$$

Substituting the values of the parametric constants Equations (42) to (50) into Equations (3) and (4), respectively, one gets the energy eigenvalues and the corresponding unnormalized radial eigenstates as

$$
E_{n l}=-\frac{\hbar^{2} \alpha^{2}}{2 \mu}\left[\frac{n(n+1)+l(l+1)+\frac{1}{2}+(n+1) T-\frac{2 \mu C}{\hbar^{2} \alpha^{2}}}{1+2 n+T}\right]^{2},
$$


and

$$
\psi_{n l}(r)=N_{n l} \mathrm{e}^{-\alpha \sqrt{\gamma_{3}} r}\left(1-\mathrm{e}^{-\alpha r}\right)^{\beta} P_{n}^{\left(2 \sqrt{\gamma_{3}}, T\right)}\left(1-2 \mathrm{e}^{-\alpha r}\right),
$$

where

$$
\begin{gathered}
T=\sqrt{(2 l+1)^{2}-\frac{8 \mu}{\hbar^{2} \alpha^{2}}\left(C+D-\alpha^{2} V_{1}\right)}, \\
\gamma_{3}=-\frac{2 \mu E}{\hbar^{2} \alpha^{2}}
\end{gathered}
$$

and $\beta=\frac{1+T}{2}-\sqrt{\gamma_{3}}$.

\subsection{Special Cases}

To get special cases some parameters should be set to zero. The first case is Manning-Rosen plus Mobius square which can be obtained by setting $V_{1}$ to zero and the energy eigenvalues will be

$$
E_{n l}=-V_{0} A^{2}-\frac{\alpha^{2} \hbar^{2}}{2 \mu}\left[\frac{n(n+1)+l(l+1)+0.5+T(n+1)-\frac{2 \mu}{\alpha^{2} \hbar^{2}}\left(C+2 A V_{0}(A+B)\right)}{1+2 n+T}\right]^{2}
$$

where

$$
T=\sqrt{(2 l+1)^{2}-\frac{8 \mu}{\hbar^{2} \alpha^{2}}\left(C+D+(A+B)^{2} V_{0}\right)} .
$$

The second case is Maning-Rosen plus quadratic Yukawa potential which can be obtained by setting $V_{0}$ to zero. The eigenvalues obtained are as follows

$$
E_{n l}=-\frac{\alpha^{2} \hbar^{2}}{2 \mu}\left[\frac{n(n+1)+l(l+1)+0.5+T(n+1)-\frac{2 \mu C}{\alpha^{2} \hbar^{2}}}{1+2 n+T}\right]^{2}
$$

where

$$
T=\sqrt{(2 l+1)^{2}-\frac{8 \mu}{\hbar^{2} \alpha^{2}}\left(C+D-\alpha^{2} V_{1}\right)},
$$

which is the same results as in (51) and (53).

The third case is Mobius square plus inversely quadratic Yukawa potential, which results from substituting $C=D=0$ in (17). The eigenvalues resulting from substituting these parameters in (55) are given by [38]

$$
E_{n l}=-V_{0} A^{2}-\frac{\hbar^{2} \alpha^{2}}{2 \mu}\left[\frac{n(n+1)+l(l+1)+\frac{1}{2}+(n+1) T-\frac{2 \mu}{\hbar^{2} \alpha^{2}}\left(2 V_{0} A(A+B)\right)}{1+2 n+T}\right]^{2},
$$

where

$$
T=\sqrt{(2 l+1)^{2}-\frac{8 \mu}{\hbar^{2} \alpha^{2}}\left((A+B)^{2} V_{0}-\alpha^{2} V_{1}\right)}
$$


The fourth case is Manning-Rosen which can be obtained by substituting $V_{1}=V_{0}=0$ and the resulting eigenvalues are given as

$$
E_{n l}=-\frac{\hbar^{2} \alpha^{2}}{2 \mu}\left[\frac{n(n+1)+l(l+1)+\frac{1}{2}+(n+1) T-\frac{2 \mu C}{\hbar^{2} \alpha^{2}}}{1+2 n+T}\right]^{2}
$$

where

$$
T=\sqrt{(2 l+1)^{2}-\frac{8 \mu}{\hbar^{2} \alpha^{2}}(C+D)},
$$

which agrees with the results in [39] and [40].

The fifth case is Mobius square potential which can be obtained by substituting $C=D=V_{1}=0$. The eigenvalues resulting are given by [19]

$$
E_{n l}=-V_{0} A^{2}-\frac{\hbar^{2} \alpha^{2}}{2 \mu}\left[\frac{n(n+1)+l(l+1)+\frac{1}{2}+(n+1) T-\frac{2 \mu}{\hbar^{2} \alpha^{2}}\left(2 V_{0} A(A+B)\right)}{1+2 n+T}\right]^{2},
$$

where

$$
T=\sqrt{(2 l+1)^{2}-\frac{8 \mu}{\hbar^{2} \alpha^{2}}\left((A+B)^{2} V_{0}\right)} .
$$

The sixth case is inversely quadratic Yukawa potential which can be obtained by setting $C=D=V_{0}=0$ and the eigenvalues produced are given by [41]

$$
E_{n l}=-\frac{\hbar^{2} \alpha^{2}}{2 \mu}\left[\frac{n(n+1)+l(l+1)+\frac{1}{2}+(n+1) T}{1+2 n+T}\right]^{2}
$$

where

$$
T=\sqrt{(2 l+1)^{2}+\frac{8 \mu V_{1}}{\hbar^{2}}}
$$

The seventh case is the Hulthèn potential which can be obtained by substituting $D=-C$ in the Manning-Rosen Potential and the resulting potential will be [42]

$$
V(r)=-\frac{C \mathrm{e}^{-\alpha r}}{1-\mathrm{e}^{-\alpha r}}
$$

and the eigenvalues obtained by substituting the parameters in (31) will be

$$
E_{n l}=-\frac{\hbar^{2} \alpha^{2}}{2 \mu}\left[\frac{n(n+1)+l(l+1)+\frac{1}{2}+(n+1)(2 l+1)-\frac{2 \mu C}{\hbar^{2} \alpha^{2}}}{2(1+n+l)}\right]^{2},
$$

which is the same results as in [43] and [44]. 


\section{Numerical Results and Discussion}

In this work, we have studied the solution of the Schrödinger wave equation with two sets of potentials. Here we tend to explain our results by commenting on the plotted Figures. In Figure 1, we showed the variation in the vibrational energy levels against the screening parameter $\alpha$. It is noted that as $\alpha$ increases, the energy levels of the system decrease monotonically from zero. It is equally seen that rotational energy levels of the system decrease as the screening parameter increases as shown in Figure 2. Figure 3 shows the energy states decrease as the principal quantum number increases for various values of the screening parameter.

Figure 4 indicates the variation of the vibrational energy levels against the strength parameter $D$. A decrease in the strength $D$ results in an increase in the energy. Moreover, as the potential strength $D$ decreases beyond some value it results in a sharp decrease in the energy.

In Figure 5, we examined the variation in the energy against the potential strength $V_{1}$. It is seen that the energy of the system decreases monotonically from zero as the potential strength increases for various values of $n$. A reverse case is shown in Figure 6 when energy is plotted against the potential strength $C$. It is obvious that when a particle is subjected to this system, the particle exhibits different features of $V_{1}$ and $C$ for various values of screening parameters; namely, $\alpha=0.1, \alpha=0.2$. and $\alpha=0.3$. However, when the strength parameter $C$ gets a large value, the energy drops sharply for $\alpha=0.1$.

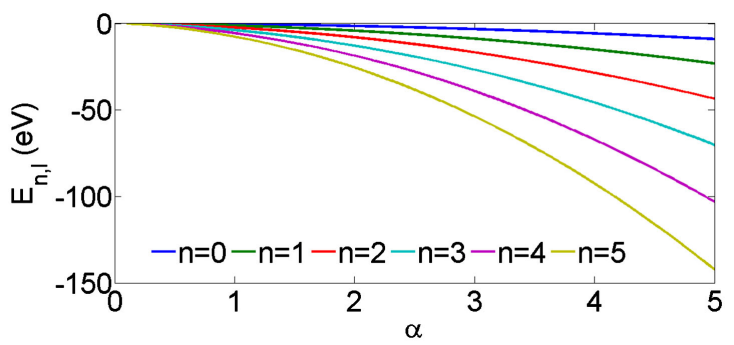

Figure 1. The quantized energy eigenvalues of Schrödinger equation with Manning-Rosen plus quadratic Yukawa potential vs $\alpha$ for different values of $n . V_{1}=0.5 \mathrm{eV}, D=-5 \mathrm{eV}$, $C=0.5 \mathrm{eV}$ and $l=0$.

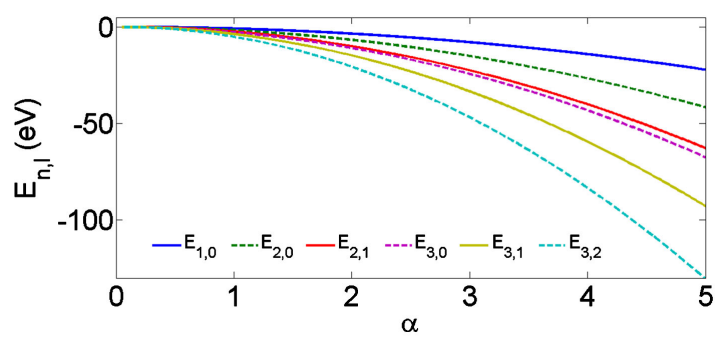

Figure 2. The quantized energy eigenvalues of Schrödinger equation with Manning-Rosen plus quadratic Yukawa potential vs $\alpha . V_{1}=0.5 \mathrm{eV}, D=-5 \mathrm{eV}$, and $C=0.5 \mathrm{eV}$ for $n=1 \quad(l=0), n=2 \quad(l=0, l=1)$, and $n=3 \quad(l=0, l=1, l=2)$. 


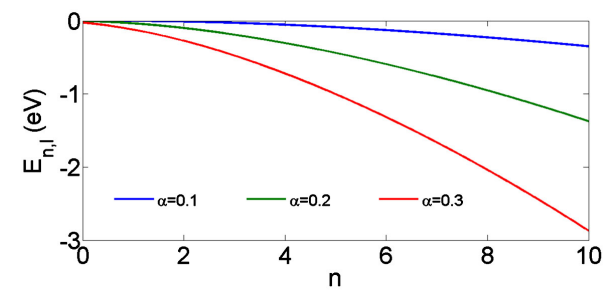

Figure 3. The quantized energy eigenvalues of Schrödinger equation with Manning-Rosen plus quadratic Yukawa potential vs $n . V_{1}=0.5 \mathrm{eV}, D=-5 \mathrm{eV}, C=0.5 \mathrm{eV}$ and $l=0$ for different values of $\alpha$.

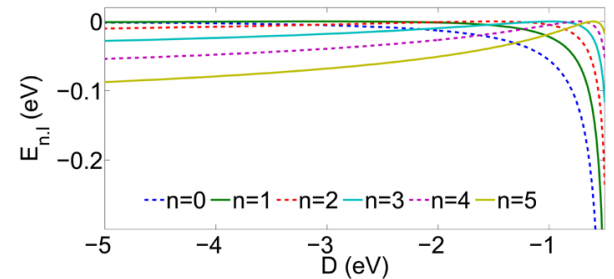

Figure 4. The quantized energy eigenvalues of Schrödinger equation with Manning-Rosen plus quadratic Yukawa potential vs $D . V_{1}=0.5 \mathrm{eV}, \alpha=0.1, C=0.5 \mathrm{eV}$ and $l=0$ for different values of $n$.

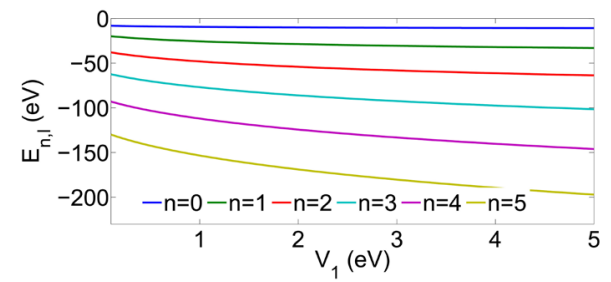

Figure 5. The quantized energy eigenvalues of Schrödinger equation with Manning-Rosen plus quadratic Yukawa potential vs $V_{1} . D=-5 \mathrm{eV}, \alpha=0.1, C=0.5 \mathrm{eV}$ and $l=0$ for different values of $n$.

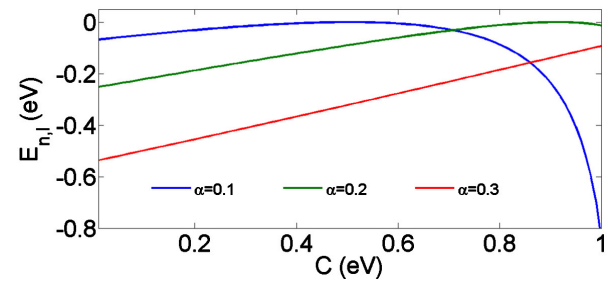

Figure 6. The quantized energy eigenvalues of Schrödinger equation with Manning-Rosen plus quadratic Yukawa potential vs $C$. $D=-5 \mathrm{eV}, V_{1}=0.5 \mathrm{eV}, n=0$ and $l=0$ for different values of $\alpha$.

Figure 7 shows a plot of the variation in the energy against the strength parameter $C$ for various values of $n$. It is seen that the energy decreases as the strength parameter $C$ increases. It is equally seen that the vibrational energy of the system decreases as the screening parameter increases for various values of $n$ as shown in Figure 8. A similar behavior to Figure 8 appears for the rotational energy levels when plotted against the screening parameters are shown in Figure 9. 
Finally, Figure 10 shows the decrease of vibrational energy levels as the quantum number $n$ increases for various values of screening parameter $\alpha$.

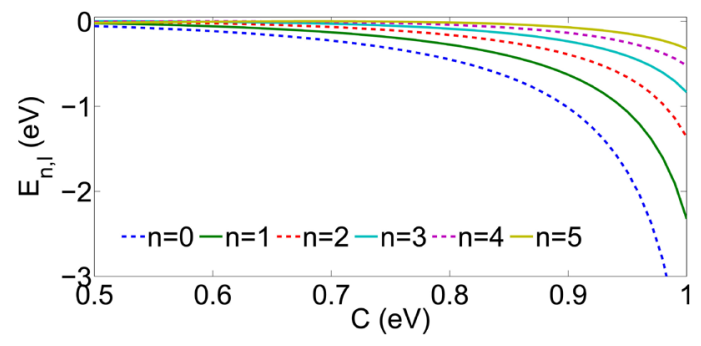

Figure 7. The quantized energy eigenvalues of Schrödinger equation with Manning-Rosen plus quadratic Yukawa potential vs $C . D=-5 \mathrm{eV} \quad V_{1}=0.5 \mathrm{eV}, \alpha=0.1$ and $l=0$ for different values of $n$.

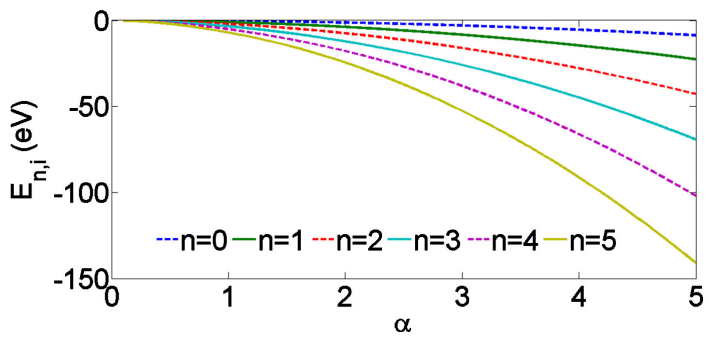

Figure 8. The quantized energy eigenvalues of Schrödinger equation with ManningRosen Mobius square plus quadratic Yukawa potential vs $\alpha . D=-5 \mathrm{eV}, V_{1}=0.5 \mathrm{eV}$, $C=0.5 \mathrm{eV}$ and $l=0$ for different values of $n$.

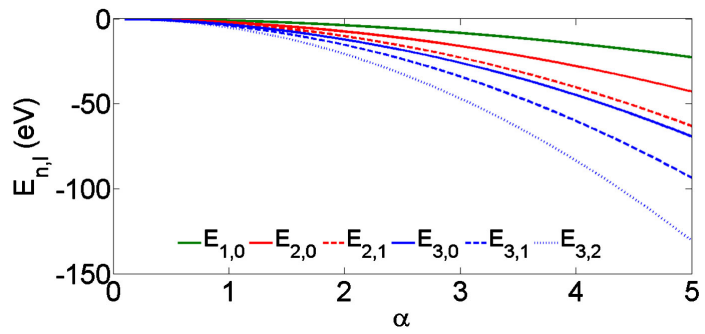

Figure 9. The quantized energy eigenvalues of Schrödinger equation with Manning-Rosen Mobius square plus quadratic Yukawa potential vs $\alpha . D=-5 \mathrm{eV}, V_{1}=0.5 \mathrm{eV}$ and $C=0.5 \mathrm{eV}$ for different values of $n$.

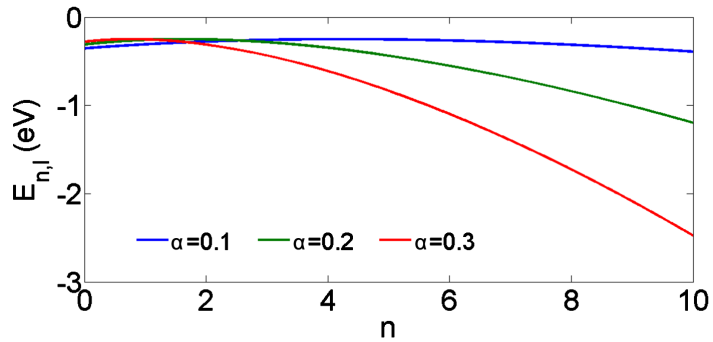

Figure 10. The quantized energy eigenvalues of Schrödinger equation with Manning-Rosen Mobius square plus quadratic Yukawa potential vs $n . D=-5 \mathrm{eV}, V_{1}=0.5 \mathrm{eV}, C=0.5 \mathrm{eV}$ and $l=0$ for different values of $\alpha$. 


\section{Conclusion}

In this work, we have analytically solved the non-relativistic wave equation with the combination of three important potentials via the parametric Nikiforov-Uvarov method. We have obtained the energy equation and the corresponding non-normalized wave functions of the combination set of Manning-Rosen plus Mobius square plus quadratic Yukawa potential and their subset of potentials. We have obtained in detail the energy eigenvalues and the corresponding wave function for subset of potentials. These results could find their applications in atomic as well as molecular physics. The effects of the strength parameters as well as screening parameter on the vibrational and rotational energy levels were also studied.

\section{Acknowledgements}

We thank the Editor and the referees for their valuable comments. This generous support is greatly appreciated.

\section{Conflicts of Interest}

The authors declare no conflicts of interest regarding the publication of this paper.

\section{References}

[1] Hou, C.-F., Zhou, Z.-X. and Li, Y. (1999) Bound States of the Klein-Gordon Equation with Vector and Scalar Wood-Saxon Potentials. Acta Physica Sinica, 8, 561-564. https://doi.org/10.1088/1004-423X/8/8/001

[2] Ikhdair, S.M. and Sever, R. (2008) Polynomial Solutions of the Mie-Type Potential in the $D$-Dimensional Schrödinger Equation. Journal of Molecular Structure THEOCHEM, 855, 13-17. https://doi.org/10.1016/j.theochem.2007.12.044

[3] Sever, R., Tezcan, C. and Yesiltas, O. (2008) Exact Solution of Effective Mass Schrödinger Equation for the Hulthen Potential. International Journal of Theoretical Physics, 47, 2243-2248. https://doi.org/10.1007/s10773-008-9656-7

[4] Dong, S. and Dong, S.H. (2002) Schrödinger Equation with a Coulomb Field in 2+1 Dimensions. Physica Scripta, 66, 342-344. https://doi.org/10.1238/Physica.Regular.066a00342

[5] de Souza-Dutra, A. (1993) Conditionally Exactly Soluble Class of Quantum Potentials. Physical Review A, 47, Article No. R2435. https://doi.org/10.1103/PhysRevA.47.R2435

[6] Alhaidari, A.D. (2007) Representation Reduction and Solution Space Contraction in Quasi-Exactly Solvable Systems. Journal of Physics A: Mathematical and Theoretical, 40, 6305-6328. https://doi.org/10.1088/1751-8113/40/24/004

[7] Bander, M. and Itzykson, C. (1968) Group Theory and the Hydrogen Atom (I). Reviews of Modern Physics, 38, 330-345. https://doi.org/10.1103/RevModPhys.23.21

[8] Infeld, L. and Hull, T.D. (1951) The Factorization Method. Reviews of Modern Physics, 23, 21-68. https://doi.org/10.1103/RevModPhys.23.21

[9] Dong, S.H. (2007) Factorization Method in Quantum Mechanics. Springer, Amsterdam, 150-155. https://doi.org/10.1007/978-1-4020-5796-0 
[10] Jia, C.S. and Jia, Y. (2017) Relativistic Rotation-Vibrational Energies for the $\mathrm{Cs}_{2}$ molecule. The European Physical Journal D, 71, Article No. 3. https://doi.org/10.1140/epjd/e2016-70415-y

[11] Junker, G. (1998) Supersymmetric Methods in Quantum and Statistical Physics. Springer-Verlag, Berlin, Heidelberg.

[12] Setare, M.R. and Nazari, Z. (2009) Solution of Dirac Equations with Five-Parameter Exponent-Type Potential. Acta Physica Polonica Series B, 40, 2809-2824.

[13] Nikiforov, A.F. and Uvarov, V.B. (1988) Special Functions of Mathematical Physics. Birkhäuser, Basel, 205. https://doi.org/10.1007/978-1-4757-1595-8

[14] Qiang, W.C., Gao, Y. and Zhou, R.S. (2008) Arbitrary/-State Approximate Solutions of the Hulthén Potential through the Exact Quantization Rule. Central European Journal of Physics, 6, 356-362. https://doi.org/10.2478/s11534-008-0041-1

[15] Ikhdair, S.M. and Sever, R. (2009) Exact Quantization Rule to the Kratzer-Type Potentials: An Application to the Diatomic Molecules. Journal of Mathematical Chemistry, 45, Article No. 1137. https://doi.org/10.1007/s10910-008-9438-8

[16] Hamzavi, H., Rajabi, A.A. and Hassanabadi, H. (2010) Exact Pseudospin Symmetry Solution of the Dirac Equation for Spatially-Dependent Mass Coulomb Potential Including a Coulomb-Like Tensor Interaction via Asymptotic Iteration Method. Physics Letters A, 374, 4303-4307. https://doi.org/10.1016/j.physleta.2010.08.065

[17] Ita, B.I., Louis, H., Orosun, M.M., Nzeata-Ibe, N., Ikeuba, A.I. and Magu, T.O. (2018) Radial Solutions of the Schrodinger's Equation with Superposition of Yukawa and Manning-Rosen Applying the Nikiforov-Uvarov Method. Communications in Applied Sciences, 6, 1-10.

[18] Zhang, M.-C. and An, B. (2010) Analytical Solutions of the Manning-Rosen Potential in the Tridiagonal Program. Chinese Physics Letters, 27, Article ID: 110301. https://doi.org/10.1088/0256-307X/27/11/110301

[19] Okorie, U.S., Ikot, A.N., Onyeaju, M.C. and Chukwuocha, E.O. (2018) Bound State Solutions of Schrödinger Equation with Modified Mobius Square Potential (MMSP) and Its Thermodynamic Properties. Journal of Molecular Modeling, 24, Article No. 289. https://doi.org/10.1007/s00894-018-3811-8

[20] Onate, C.A. and Ojonubah, J.O. (2016) Eigensolutions of the Schrödinger Equation with a Class of Yukawa Potentials via Supersymmetric Approach. Journal of Theoretical and Applied Physics, 10, 21-26. https://doi.org/10.1007/s40094-015-0196-2

[21] Ikot, A.N., Zarrinkamar, S., Ibanga, E.J., Maghsoodi, E. and Hassanabadi, H. (2014) Pseudospin Symmetry of the Dirac Equation for a Möbius Square Plus Mie Type Potential with a Coulomb-Like Tensor Interaction via SUSYQM. Chinese Physics $C$, 38, Article ID: 013101. https://doi.org/10.1088/1674-1137/38/1/013101

[22] Maghsoodi, E., Hassanabadi, H., Rahimov, H. and Zarrinkamar, S. (2013) Arbitrary-State Solutions of the Dirac Equation for a Möbius Square Potential Using the Nikiforov-Uvarov Method. Chinese Physics C, 37, Article ID: 043105. https://doi.org/10.1088/1674-1137/37/4/043105

[23] Yazarloo, B.H., Hassanabadi, H. and Zarrinkamar, S. (2012) Oscillator Strengths Based on the Möbius Square Potential under Schrödinger Equation. The European Physical Journal Plus, 127, Article No. 51. https://doi.org/10.1140/epjp/i2012-12051-9

[24] Ikot, A.N., Hassanabadi, H., Maghsoodi, E. and Yazarloo, B.H. (2014) Bound and Scattering States of Modified Yukawa Potential under Relativistic Spin and Pseudospin Symmetries with Three Tensor Interactions. The European Physical Journal Plus, 129, Article No. 218. https://doi.org/10.1140/epjp/i2014-14218-8 
[25] Ikot, A.N, Maghsoodi, E., Zarrinkamar, S. and Hassanabadi, H. (2013) Relativistic Spin and Pseudospin Symmetries of Inversely Quadratic Yukawa-Like Plus Mobius Square Potentials Including a Coulomb-Like Tensor Interaction. Few-Body Systems, 54, 2027-2040. https://doi.org/10.1007/s00601-013-0701-6

[26] Ikot, A.N., Obong, H.P. and Hassanabadi, H. (2015) Relativistic Symmetries of (D+1) Dimensional Dirac Equation with Multiparameter Exponential-Type Potentials Using Supersymmetric Quantum Mechanics. Few-Body Systems, 56, 185-196. https://doi.org/10.1007/s00601-015-0974-Z

[27] Antia, A.D., Ikot, A.N., Hassanabadi, H. and Maghsoodi, E. (2013) Bound State Solutions of Klein-Gordon Equation with Mobius Square Plus Yukawa Potentials. Indian Journal of Physics, 87, 1133-1139. https://doi.org/10.1007/s12648-013-0336-y

[28] Farout, M., Sever, R. and Ikhdair, S.M. (2020) Approximate Solution to the TimeDependent Kratzer Plus Screened Coulomb Potential in the Feinberg-Horodecki Equation. Chinese Physics B, 29, Article ID: 060303.

https://doi.org/10.1088/1674-1056/ab8379

[29] Farout, M. and Ikhdair, S.M. (2020) Momentum Eigensolutions of Feinberg-Horodecki Equation with Time-Dependent Screened Kratzer-Hellmann Potential. Journal of Applied Mathematics and Physics, 8, 1207-1221.

https://doi.org/10.4236/jamp.2020.87091

[30] Farout, M., Bassalat, A. and Ikhdair, S.M. (2020) Exact Quantized Momentum Eigenvalues and Eigenstates of a General Potential Model. Journal of Applied Mathematics and Physics, 8, 1434-1447. https://doi.org/10.4236/jamp.2020.87109

[31] Farout, M., Bassalat, A. and Ikhdair, S.M. (2020) Feinberg-Horodecki Exact Momentum States of Improved Deformed Exponential-Type Potential. Journal of Applied Mathematics and Physics, 8, 1496-1506. https://doi.org/10.4236/jamp.2020.88115

[32] Tezcan, C. and Sever, R. (2009) A General Approach for the Exact Solution of the Schrödinger Equation. International Journal of Theoretical Physics, 48, 337-350. https://doi.org/10.1007/s10773-008-9806-y

[33] Ikot, A.N. (2012) Solutions to the Klein-Gordon Equation with Equal Scalar and Vector Modified Hylleraas Plus Exponential Rosen Morse Potentials. Chinese Physics Letters, 29, Article ID: 060307. https://doi.org/10.1088/0256-307X/29/6/060307

[34] Ahmaadov, A.I., Demirci, M. and Aslanova, S.M. (2019) Bound State Solutions of the Klein-Fock-Gordon Equation with the Sum of Manning-Rosen Potential and Yukawa Potential within SUSYQM. 26th International Conference on Integrable Systems and Quantum Symmetries, Prague, 8-12 July 2019, Article ID: 012001. https://doi.org/10.1088/1742-6596/1416/1/012001

[35] Edet, C.O., Okoi, P.O. and Chima, S.O. (2020) Analytic Solutions of the Schrödinger Equation with Non-Central Generalized Inverse Quadratic Yukawa Potential. Revista Brasileira de Ensino de Física, 42, Article ID: e20190083.

https://doi.org/10.1590/1806-9126-rbef-2019-0083

[36] Ikot, A.I., Maghsoodi, E., Antia, A.D., Zarrinkamar, S. and Hassanabadi, H. (2013) Approximate $\kappa$-State Solutions to the Dirac Mobius Square-Yukawa and Mobius square-Quasi Yukawa Problems under Pseudospin and Spin Symmetry Limits with Coulomb-Like Tensor Interaction. Canadian Journal of Physics, 91, 560-575. https://doi.org/10.1139/cjp-2012-0506

[37] Greene, R.L. and Aldrich, C. (2013) Variational Wave Functions for a Screened Coulomb Potential. Physical Review A, 14, 2363-2366. 
https://doi.org/10.1103/PhysRevA.14.2363

[38] Antia, A.D., Essien, I.E., Umoren, E.B. and Eze, C.C. (2015) Approximate Solutions of the Non-Relativistic Schrödinger Equation with Inversely Quadratic Yukawa Plus Mobius Square Potential via Parametric Nikiforov-Uvarov Method. Advances in Physics Theories and Applications, 44, 1-13.

[39] Falaye, B.J., Oyewumi, K.J., Ibrahim, T.T., Punyasena, M.A. and Onate, C.A. (2013) Bound State Solutions of the Manning-Rosen Potential. Canadian Journal of Physics 91, 98-104. https://doi.org/10.1139/cjp-2012-0330

[40] Ikhdair, S.M. (2011) On the Bound-State Solutions of the Manning-Rosen Potential Including an Improved Approximation to the Orbital Centrifugal Term. Physica Scripta, 83, Article ID: 015010. https://doi.org/10.1088/0031-8949/83/01/015010

[41] Ita, I. B., Ikeuba, A.I., Louis, H. and Tchoua, P. (2015) Solutions of the Schrödinger Equation with Inversely Quadratic Yukawa plus Attractive Radial Potential Using Nikiforov-Uvarov Method. AIP Conference Proceedings, 1629, 360-362. https://doi.org/10.1063/1.4902294

[42] Jia, C.S., Liu, J.Y. and Wang, P.Q. (2008) A New Approximation Scheme for the Centrifugal Term and the Hulthén Potential. Physics Letters A, 372, 4779-4782. https://doi.org/10.1016/j.physleta.2008.05.030

[43] Ikhdair, S.M. (2009) An Improved Approximation Scheme for the Centrifugal term and the Hulthén Potential. The European Physical Journal A, 39, 307-314. https://doi.org/10.1140/epja/i2008-10715-2

[44] Ikhdair, S.M. and Abu-Hasna, J. (2011) Quantization Rule Solution to the Hulthén Potential in Arbitrary Dimension with a New Approximate Scheme for the Centrfugal Term. Physica Scripta, 83, Article ID: 025002.

https://doi.org/10.1088/0031-8949/83/02/025002 